\title{
Effect of Metal Oxide Surge Arrester on Ferroresonance in Voltage Transformer Including Nonlinear Core Losses
}

\author{
Hamid Radmanesh, Mehrdad Rostami, and Jafar Khalilpour
}

\begin{abstract}
This paper studies the effect of Metal Oxide Surge Arrester (MOSA) on ferroresonance overvoltage transformer (VT) considering nonlinear core losses effect. It is expected that MOSA generally cause ferroresonance 'dropout'. Time-domain study has been done to study this effect. Simulation has been done on a voltage transformer rated $100 \mathrm{VA}, 275 \mathrm{kV}$. The magnetization characteristic of the transformer is modelled by a single-value two-term polynomial with order seven. The core loss is modelled by nonlinear resistance. The simulation results reveal that connecting the MOSA in parallel to the VT exhibits a great controlling effect on ferroresonance overvoltages. Phase plane, voltage waveforms, along with bifurcation diagrams are also derived. Significant effect on the onset of chaos, the range of parameter values that may lead to chaos along with ferroresonance overvoltages has been obtained and presented.
\end{abstract}

Index Terms-Metal oxide surge arrestor, nonlinear core losses chaos, bifurcation, ferroresonance, voltage transformers

\section{INTRODUCTION}

Ferroresonance occurs when a nonlinear inductor, usually a transformer with a saturable magnetic core, is excited through a linear capacitor from a sinusoidal source, particularly in the presence of long lines or capacitive power cables. It is usually initiated by a system disturbance of some form, for example, the disconnection of transformer feeder lines or the opening of a circuit breaker in series with a voltage transformer. Also it can produce unpredictable overvoltages and abnormal currents. The prerequisite for ferroresonance is a circuit containing nonlinear iron core inductance and some existed capacitance. The abrupt transition or jump from one state to another is triggered by a disturbance, switching action or a gradual change in values of a parameter. The first analytical work was done by Rudenberg in the 1940's [1]. More exacting and detailed work was done later by Hayashi in the 1950's [2]. Subsequent research has been divided into two main areas: improving the transformer models and studying ferroresonance at the system level. Typical cases of ferroresonance are reported in [3]. Effect of circuit breaker grading capacitance on ferroresonance in VT is investigated in [4]. There are a number of cases of ferroresonance and a typical case is described in [5]. Frame [6] and others have

Manuscript received September 9, 2012; revised October 23, 2012.

Hamid Radmanesh and Jafar Khalilpour are with the Electrical Engineering Department, Aeronautical University of Science\& Technology, Tehran, Iran (e-mail: Hamid.radmanesh@aut.ac.ir, J_khalilpour@yahoo.com).

Mehrdad Rostami is with Electrical Engineering Department, Shahed University, End of Khalij-e-Fars High way, In front of Imam Khomeini holly shrine, Tehran-1417953836 , Iran (e-mail: rostami@shahed.ac.ir). developed piecewise-linear methods of modelling the nonlinearities in saturable inductances. Hopkinson [5] performed system tests and simulations on the effect of different switching strategies on the initiation of ferroresonance in three phase systems. Smith [7] categorized the modes of ferroresonance in one type of three phase distribution transformer based on the magnitude and appearance of the voltage waveforms. Arturi [8] and Mork [9] have demonstrated the use of duality transformations to obtain transformer equivalent circuits. In [10], the chaotic behaviour of the simple power system is investigated for a range of loading conditions through computer simulations. The analysis of the severe overvoltages caused by neutral shift and ferroresonance due to the disconnection of one phase of an ungrounded-ye delta transformer bank from the source is presented in [11]. The implications of applying MOSA in the distribution environment are described in [12], [13]. In [14], the performances of MOSA exposed to ferroresonance conditions in pad mount transformers are analyzed. The effect of a connected MOSA in parallel to the power transformer is illustrated in [15]. Evaluation of chaotic ferroresonance in power transformers including nonlinear core losses is investigated in [16]. Analysis of ferroresonance puenomena in power transformers including neutral resistance effect is investigated in [17]. Finally effect of circuit breaker shunt resistance on chaotic ferroresonance in voltage transformer has shown in [18], in this work ferroresonance has been controlled by considering C.B resistance effect. In all previous studies, the effect of MOSA on ferroresonance puenomena in voltage transformer has been neglected. Current paper studies the effect of MOSA on the global behaviour of a VT ferroresonance circuit in voltage transformer while its core losses have a nonlinear behaviour.

\section{System Modeling Without MOSA}

During VT ferroresonance an oscillation occurs between the nonlinear iron core inductance of the VT and existing capacitances of network. In this case, energy is coupled to the nonlinear core of the voltage transformer via the open circuit breaker grading capacitance or system capacitance to sustain the resonance. The result may be saturation in the VT core and very high voltage up to 4pu can theoretically gained in worst case conditions. The magnetizing characteristic of a typical 100VA VTs can be presented by 7 order polynomial [18]. Fig. 1 shows the single line diagram of the most commonly encountered system arrangement that can give rise to VT ferroresonance [18]. Ferroresonance can occur upon opening of disconnector 3 with circuit breaker open and 
either disconnector 1 or 2 closed. Alternatively it can also occur upon closure of both disconnections 1 or 2 with circuit breaker and disconnector 3 open [18].

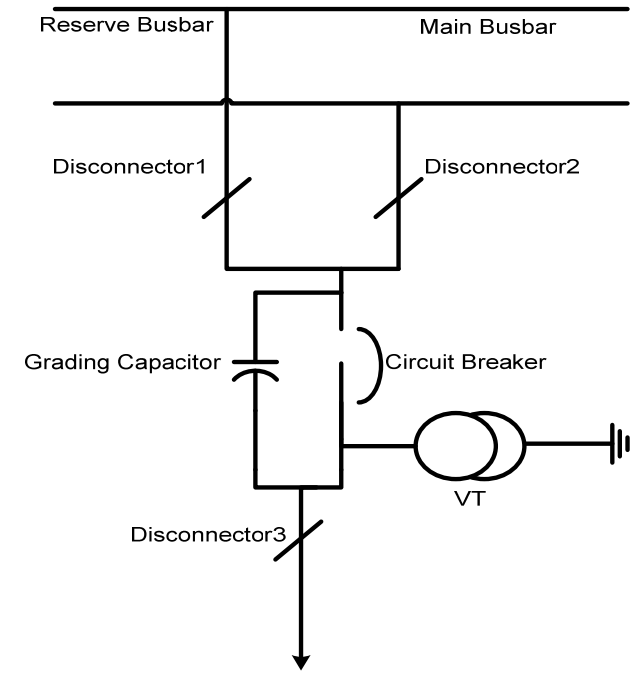

Fig. 1. System one line diagram arrangement resulting to VT ferroresonance

The system arrangement shown in Fig. 1 can effectively be reduced to an equivalent circuit as shown in Fig. 2.

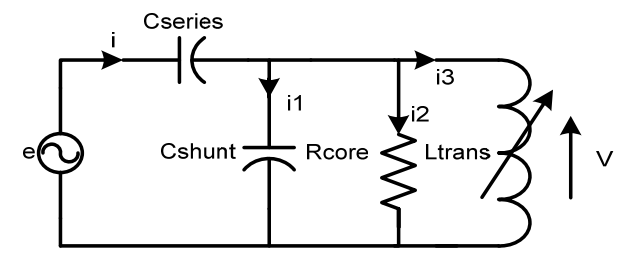

Fig. 2. Basic reduced equivalent ferroresonance circuit [30]

In Fig. 2, $E$ is the RMS supply phase voltage, $C_{\text {series }}$ is the circuit breaker grading capacitance and $C_{\text {shunt }}$ is the total phase-to-earth capacitance of the arrangement. The resistor $R$ represents a voltage transformer core loss that has been found to be an important factor in the initiation of ferroresonance. In the peak current range for steady-state operation, the flux-current linkage can be approximated by a linear characteristic such as $i_{L}=a \lambda$ where the coefficient of the linear term (a) corresponds closely to the reciprocal of the inductance ( $a \cong 1 / L)$. However, for very high currents the iron core might be driven into saturation and the flux-current characteristic becomes highly nonlinear, here the $\lambda-i$ characteristic of the voltage transformer is modeled as in [8] by the polynomial

$$
i=a \lambda+b \lambda^{7}
$$

where, $a=3.14, b=0.41$

\section{SYSTEM DYNAMIC AND EQUATION}

In this paper, the core loss model adopted is described by a third order power series which coefficients are fitted to match the hysteresis and eddy current nonlinear characteristics given in [19]:

$$
i_{R m}=h_{0}+h_{1} V_{m}+h_{2} V_{m}^{2}+h_{3} V_{m}^{3}
$$

Per unit value of $\left(i_{R m}\right)$ is given in (3)

$$
i_{R m}=-0.000001+0.0047 V_{m}-0.0073 V_{m}^{2}+0.0039 V_{m}^{3}
$$

Mathematical analysis of equivalent circuit by applying KVL and KCL has been done and equations of system can be presented as below:

$$
\begin{gathered}
\lambda_{\text {peak }}=\sqrt{2} \frac{v_{R M S}}{\omega} \\
v_{L}=\frac{d \lambda}{d t} \\
i=C_{s e r} \frac{d\left(e-v_{L}\right)}{d t}=C_{s e r}\left(\dot{e}-\frac{d^{2} \lambda}{d t^{2}}\right) \\
i=i_{1}+i_{2}+i_{3} \Rightarrow \frac{C_{s h}}{\left(C_{s e r}+C_{s h}\right)}(\sqrt{2} E \cos \omega t)=\frac{1}{\omega} \frac{d^{2} \lambda}{d t^{2}}+ \\
\frac{1}{\omega\left(C_{s e r}+C_{s h}\right)}\left(h_{0}+h_{1} v_{L}+h_{2} v_{L}^{2}+h_{3} v_{L}^{3} a \lambda+b \lambda^{7}\right)
\end{gathered}
$$

where, $\omega$ is supply frequency, and $E$ is the RMS supply phase voltage, $\mathrm{C}_{\text {series }}$ is the circuit breaker grading capacitance and $C_{\text {shunt }}$ is the total phase-to-earth capacitance of the arrangement and in (1) $a=3.4$ and $b=0.41$ are the seven order polynomial sufficient [18].

\section{Metal OXIDE Surge ArRester Model}

Surge Arrester is highly nonlinear resistor used to protect power equipment against overvoltages. The nonlinear V-I characteristic of each column of the surge arrester is modeled by combination of the exponential functions of the form:

$$
\frac{V}{V_{r e f}}=K_{i}\left(\frac{I}{I_{r e f}}\right)^{1 / \alpha_{i}}
$$

where, $V$ represents resistive voltage drop, $I$ represents arrester current and $K$ is constant and $\alpha$ is nonlinearity constant. This V-I characteristic is graphically represented as follows:

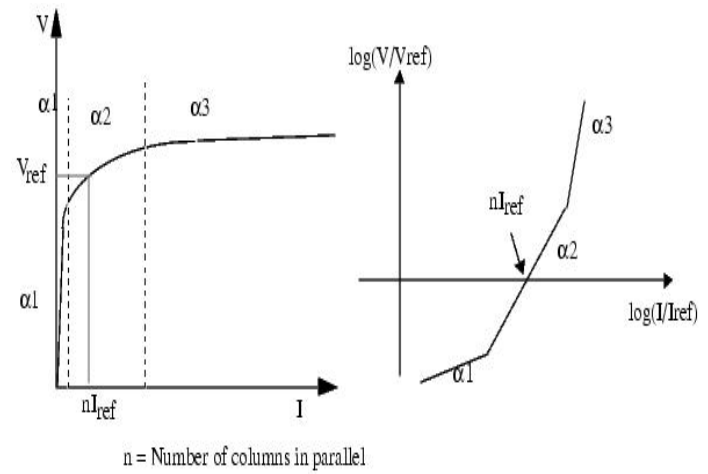

Fig. 3. V-I graphic characteristic of MOSA 


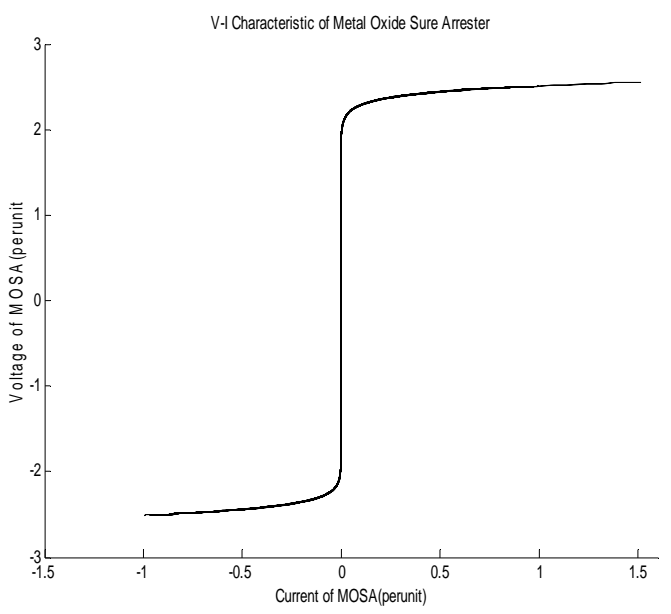

Fig. 4. V-I characteristic of MOSA, simulated diagram।

\section{SySTEM MODELING WITH MOSA}

Connecting MOSA to the system in Fig. 2, circuit can be driven in Fig. 5.

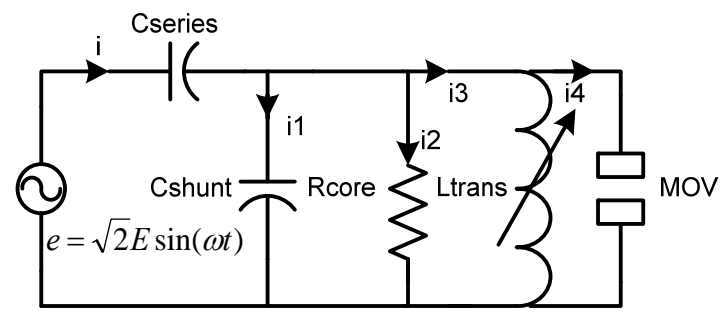

Fig. 5. Basic reduced equivalent ferroresonance circuit with MOSA

The differential equation for the circuit in Fig. 5 can be modified as follows:

$$
\begin{aligned}
& \frac{C_{s h}}{\left(C_{s e r}+C_{s h}\right)}(\sqrt{2} E \cos \omega t)=\frac{1}{\omega} \frac{d^{2} \lambda}{d t^{2}}+\frac{1}{\omega\left(C_{\text {ser }}+C_{s h}\right)} \\
& \left(h_{0}+h_{1} v_{L}+h_{2} v_{L}^{2}+h_{3} v_{L}^{3} a \lambda+b \lambda^{7}\right)+\frac{1}{C_{\text {series }}}\left(\frac{d \lambda}{k d t}\right)^{\alpha}
\end{aligned}
$$

\section{Simulation Results}

Values of $E$ and $\omega$ were fixed by 1 pu, corresponding to AC supply voltage and frequency. $C_{\text {series }}$ is the $C B$ grading capacitance and its value obviously depends on the type of circuit breaker which is used. In this analysis $C_{\text {series }}$ is assumed $0.5 \mathrm{nF}$ and $C_{\text {shunt }}$ vary between $0.1 \mathrm{nF}$ to $3 \mathrm{nF}$. Initial condition are $V(t)=\sqrt{2}, \lambda(t)=0$ at $\mathrm{t}=0$, representing circuit breaker operation at maximum voltage. In this state, system for both cases, with and without MOSA has been simulated for $\mathrm{E}=4 \mathrm{pu}$. The studied system has a periodic behavior for $\mathrm{E}=1 \mathrm{pu}$ and chaotic behavior for $\mathrm{E}=4 \mathrm{pu}$, while by applying MOSA, system behavior remain periodic for $E=1$ pu and $E=4$ pu. Figs. 6 and 7 show the simulation result for $E=4$ pu including of MOSA. By referring to Fig. 7 and comparing with Fig. 6, it is shown the MOSA clamps the overvoltages and after transient signal, its behavior reaches to the normal condition.

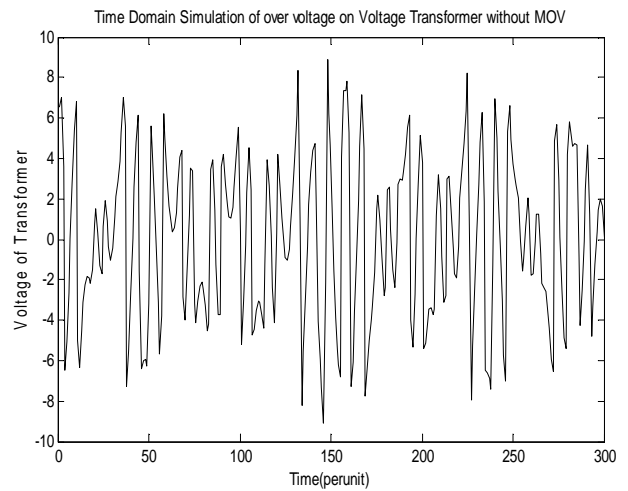

Fig. 6. Time domain simulation for chaotic motion without MOSA effect

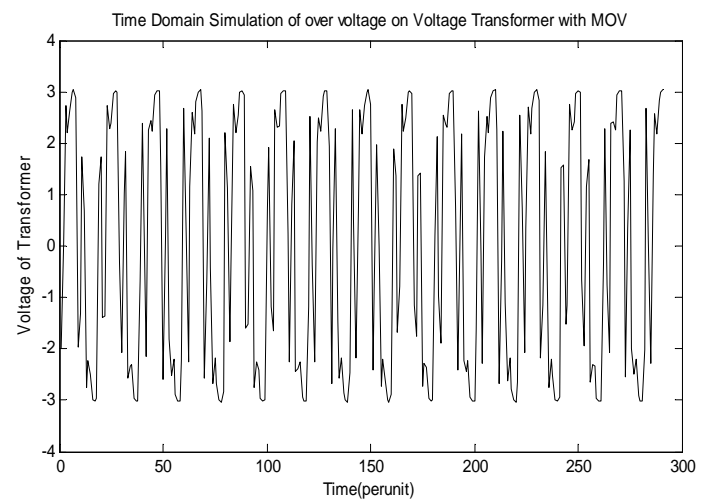

Fig. 7. Time domain simulation for chaotic motion considering MOSA effect

Corresponding phase plan diagrams has been shown the effect of the MOSA to damp the overvoltages and it is shown Figs. 8 and 9 for $E=4$ pu. It obviously shows that MOSA clamps the ferroresonance overvoltage and keep it in $\mathrm{E}=2.5$ pu. In phase plan which has been shown in Fig. 8, when the overvoltage's reaches up to $6 \mathrm{pu}$, by connecting MOSA, overvoltage's reaches to the $2.5 \mathrm{pu}$ according to the Fig. 9. It is obviously shows that MOSA clamps the ferroresonance overvoltage and keeps it in $\mathrm{E}=2.5 \mathrm{pu}$.

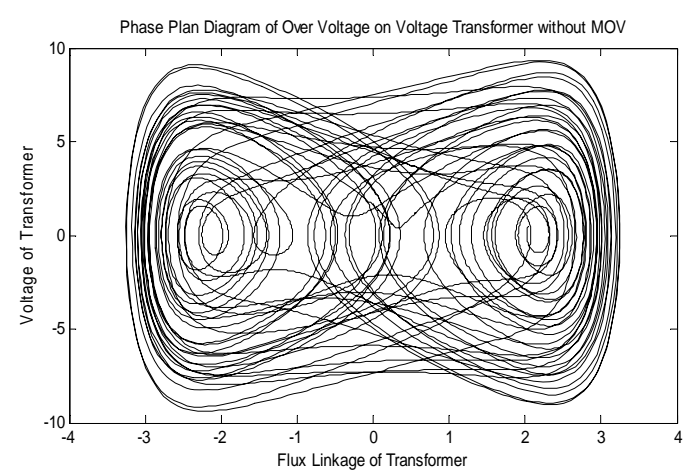

Fig. 8. Phase plan diagram for chaotic motion without MOSA effect

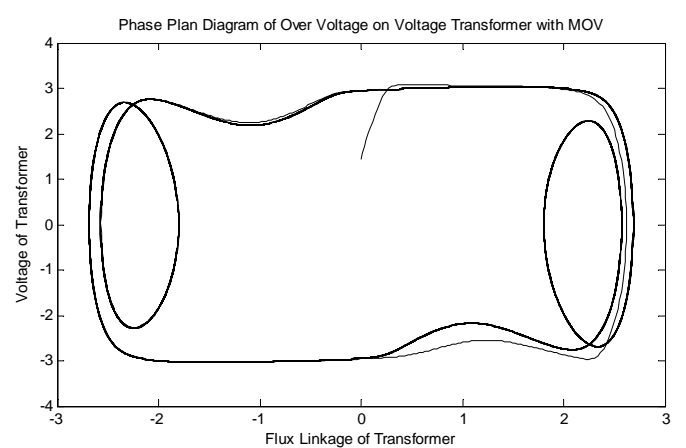

Fig. 9. Corresponding phase plan diagram considering MOSA effect 
Another tool that was used for solving the nonlinear equation of studied system is bifurcation diagram. In this paper, it is shown the effect of variation in the voltage and capacitance of the system on the ferroresonance overvoltage in the VT, and finally the effect of applying MOSA on this overvoltage by the bifurcation diagrams. By using the bifurcation diagrams, Fig. 10 clearly shows the ferroresonance overvoltage in VT when voltage of system increases up to $3 \mathrm{pu}$.

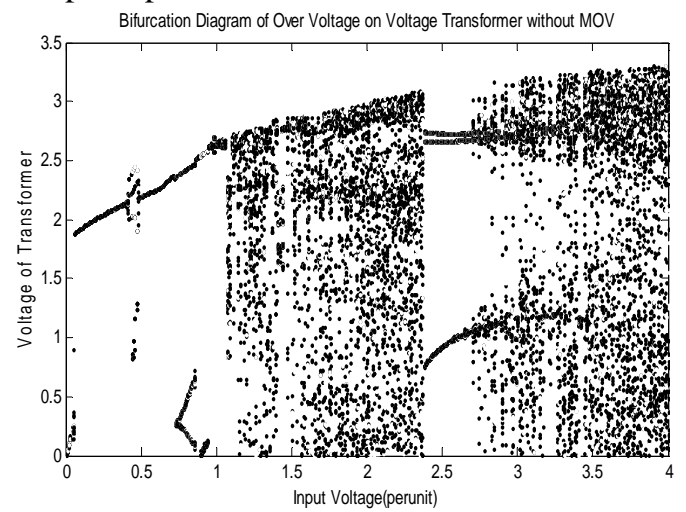

Fig. 10. Bifurcation diagram for voltage of transformer versus voltage of system, without MOSA effect

System parameters are listed in Table I.

TABLE I: PARAMETER VALUE FOR SIMULATION

\begin{tabular}{|l|c|c|}
\hline Parameter & Actual value & Per unit value \\
\hline$E$ & $275 \mathrm{kv}$ & $1 \mathrm{pu}$ \\
\hline$\omega$ & $377 \mathrm{rad} / \mathrm{sec}$ & $1 \mathrm{pu}$ \\
\hline$C_{\text {series }}$ & $0.5 \mathrm{nf}$ & $39.959 \mathrm{pu}$ \\
\hline$C_{\text {shunt }}$ & $0.1 \mathrm{nf}$ & $7.92 \mathrm{pu}$ \\
\hline & & \\
\hline
\end{tabular}

In Fig. 10 when $\mathrm{E}=0.25$ pu, voltage of VT has a period-1 behavior, in $\mathrm{E}=1 \mathrm{pu}$, period-3 appears and until $\mathrm{E}=2.5 \mathrm{pu}$ remains in the linear manner. Corresponding bifurcation diagram with the same parameter in the case of applying MOSA clearly has been shown in Fig. 11.

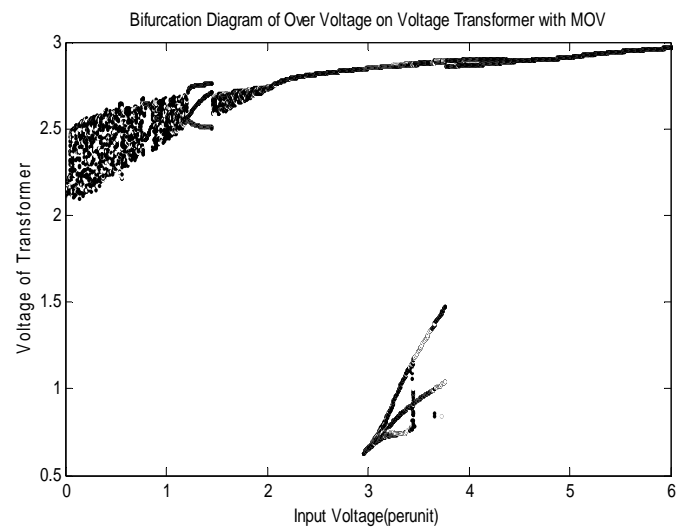

Fig. 11. Bifurcation diagram for voltage of transformer versus voltage of system considering MOSA effect

It is shown that by applying MOSA, system behaviors coming out from chaotic region and MOSA clamps the overvoltage from $8 \mathrm{pu}$ to $2.2 \mathrm{pu}$. The system behavior has period doubling bifurcation logic and there are many resonances in the system behavior. In the real systems, maximum overvoltage that VT can withstand is $4 \mathrm{pu}$, and if over voltages cross it, VT failure follows.

\section{CONCLUSION}

VTs fed through circuit breaker grading capacitance have been shown exhibiting fundamental frequency and chaotic ferroresonance conditions similar to high capacity power transformers fed via capacitive coupling from nearby sources like parallel transmission lines. Simulations have shown that a change in the value of the equivalent line to ground capacitance, may originate different types of ferroresonance overvoltages. It has also been shown that chaotic ferroresonance are not likely to occur under practical conditions but if it occurs, MOSA successfully can cause ferroresonance drop out. In the case of applying MOSA, system shows less sensitivity to initial conditions and variation in system parameters. The presence of the arrester tends to clamp the ferroresonance.

\section{ACKNOWLEDGMENT}

Corresponding author would like to appriciate Dr. Ali Nasrabadi of the Shahed University, Tehran, Iran, for providing MATLAB data files for the time domain simulations, and Mrs. Leila Kharazmi for her English editing.

\section{REFERENCES}

[1] R. Rudenberg, Transient Performance of Electric Power Systems. New York, NY: McGraw-Hill Book Company, 1950, ch. 48.

[2] C. Hayashi, Nonlinear Oscillations in Puysical Systems. New York, NY: McGraw-Hill Book Company, 1964.

[3] E. J. Dolan, D. A. Gillies, and E. W. Kimbark, "Ferroresonance in a transformer switched with an EVH line," IEEE Transactions on Power Apparatus and Systems.

[4] B. A. T. A. Zahawi, Z. Emin, and Y. K. Tong, "Chaos in ferroresonant wound voltage transformers: effect of core losses and universal circuit behavioral," IEE Proceedings*/Sci. Meas. Technol, 1998.

[5] R. H. Hopkinson, "Ferroresonance during single-phase switching of 3-phase distribution transformer banks," IEEE Trans. PAS, vol. PAS-84, no. 4, pp. 289-293, Apr. 1965.

[6] J. G. Frame, N. Mohan, and T. Liu, "Hysteresis modeling in an electromagnetic transients program,” IEEE Trans. PAS, vol. PAS-101, no. 9, pp. 3403-3411, Sept. 1982.

[7] D. R. Smith, S. R. Swanson, and J. D. Borst, "Over voltages with remotely-switched cable-fed grounded wye-wye transformers,” IEEE Trans. PAS, vol. PAS-94, no. 5, pp. 1843-1853, Sept./Oct. 1975.

[8] C. M. Arturi, 'Transient simulation and analysis of a five-limb generator step-up transformer following an out-of-phase synchronization,” IEEE Trans. Power Delivery, vol. 6, no. 1, pp. 196-207, Jan. 1991.

[9] "Ferroresonance and chaos-Observation and simulation of ferroresonance in a five-legged core distribution transformer," North Dakota State University, Pu.D. dissertation, Publication no. 9227588, UMI Publishing Services, Ann Arbor, MI 48106, (800) 521-0600, May 1992.

[10] H. D. Chiang, H. D. Liu, C. W. Variya, P. P. Wu, F. F. Lauby and, M.G, "Chaos in a simple power system," IEEE Trans, Power Syst, 1993, vol. 8, no. 940, pp. 1407-1417.

[11] R. A. Walling, K. Hartanar, and W. J. Ros, "Self generated over voltages due to open-puasing of ungrounded- wye delta transformer banks,” IEEE Trans. Power Deliv, 1995, vol. 10, no. 1, pp. 526-531.

[12] T. A. Short, J. J. Burke, and R. T. Mancao, "Application of MOVs in the distribution environment,” IEEE Trans. Power Deliv, 1994, vol. 9, no. 1, pp. 293-305.

[13] S. S. Kershaw, K. B. Gaibrois, and K. B. Stump, "Applying metal-oxide surge arrester on distribution systems," IEEE Trans. Power Deliv., 1989, vol. 4, no. 1, pp. 301-307.

[14] R. A. Walling, R .K. Hartana, R.M. Reckard, M. P. Sampart, and T. R. Balgle, "Performance of metal oxide arresters exposed to ferroresonance pad mount transformer," IEEE ran. Power Deiv, 1994, vol. 9, no. 2, pp.7888-795. 
[15] K. A. Anbarri, R. Ramanujam, T. Keerthiga, and K. Kuppusamy, "Analysis of nonlinear puenomena in MOV connected Transformers," IEE Proceedings*/Generation Transmission and Distribution 148 , 2001.

[16] A. Abbasi, H. Radmanesh, M. Rostami, and H. R. Abbasi, "Evaluation of Chaotic Ferroresonance in power transformers including Nonlinear Core Losses," Southeastcon, 2009. SOUTHEASTCON '09. IEEE, pp.1-5, 5-8 March 2009.

[17] H. Radmanesh, A. Abassi, M. Rostami, "Analysis of ferroresonance puenomena in power transformers including neutral resistance effect," Southeastcon, 2009. SOUTHEASTCON '09. IEEE, pp.1-5, 5-8 March 2009.

[18] H. Radmanesh and M. Rostami, "Effect of Circuit Breaker Shunt Resistance on Chaotic Ferroresonance in Voltage Transformer," Advances in Electrical and Computer Engineering, vol. 10, no. 3, pp. 71-77, 2010.

[19] H.W. Dommel, A. Yan, R. J. O. D. Marcano, and A. B. Miliani, in: H.P Khincha(Ed.), Tutorial Course on Digital Simulation of Transients in Power Systems (Chapter 14), IISc, Bangalore, 1983,pp. 17-38.

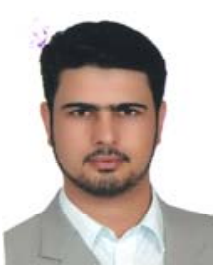

Hamid Radmanesh was born in 1981. He studied Telecommunication engineering at Malek-Ashta University of Technology, Tehran, Iran, and received the BSC degree in 2006, also studied electrical engineering at Shahed University Tehran, Iran, and received the MSC degree in 2009. Currently, He is $\mathrm{PhD}$ student in Amirkabir University of Technology. His research interests include design and modeling of power electronic converters, drives, transient and chaos in power system apparatus.

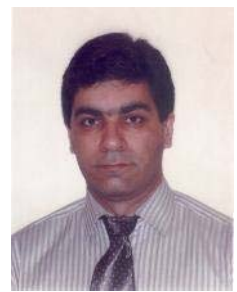

Mehrdad Rostami was born in 1965, Tehran, IRAN. $\mathrm{He}$ received BSc, MSc and Ph.D in Electrical engineering from Tehran Polytechnic University (Amir Kabir), Tehran Iran in 1988, 1991 and 2003 respectively. He is currently working as an Assistant professor and vice chancellor in research and development of Shahed University Engineering Faculty, Tehran, IRAN

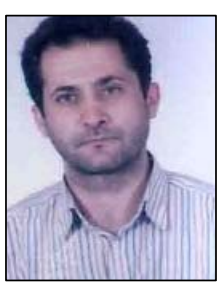

Jafaar khalilpour was born in Uremia, Iran, in 1973. He received B.S. degree from Aeronautical University of Science and Technology, Tehran, Iran, in 1995 and M.S. and Ph.D. degrees from Tarbiat Modares University, Tehran, Iran, in 1998 and 2009, all in electrical engineering. He has been an Assistant Professor with the Department of Electrical Engineering, Aeronautical University of Science and Technology, Tehran, Iran. 